\title{
Factores psicosociales, sociodemograficos, culturales y familiares asociados a Síndrome de Intestino Irritable
}

\author{
Shyrley Díaz Cárdenas ${ }^{a}$, Antonio Díaz Caballero ${ }^{\text {, }}$ \\ Katherine Margarita Arrieta Vergara ${ }^{a}$
}

a Odontóloga, Magíster en Salud Pública, Departamento de Odontología Preventiva y Social. Facultad de Odontología, Universidad de Cartagena, Colombia.

b Odontólogo, Magíster en Educación, Departamento de Medicina Oral. Facultad de Odontología, Universidad de Cartagena, Colombia.

Correspondencia: Shyrley Díaz Cárdenas, Facultad de Odontología, Universidad de Cartagena, Campus de la Salud, Departamento de Odontología Preventiva y Social, Barrio Zaragocilla, Cartagena de Indias, Colombia. Telf.:095-6698173 ext. 115, correo electrónico: shyrley77@hotmail.com.

Recibido el 15 de marzo de 2010.

Aceptado para su publicación el 2 de junio 2010.

\section{RESUMEN}

Objetivo. Describir los factores psicosociales, demográficos, culturales y familiares asociados al síndrome de intestino irritable en adultos.

Método. Estudio de casos y controles en 754 pacientes, 256 diagnosticados con Síndrome de Intestino Irritable (casos) y 498 pacientes sin la enfermedad (controles) entre los 30 y 60 años en tres instituciones privadas de salud de Cartagena de Indias seleccionados aleatoriamente, quienes no debían presentar discapacidad cognitiva, diagnostico de cáncer de colon, gástrico, páncreas, recto ni padecer alteraciones del tracto gastrointestinal. Se diligenció un cuestionario que incluyó el Test de Golberg (ansiedad y depresión), la escala de reajuste social de Holmes y Rahe (estrés) y el APGAR Familiar (funcionalidad familiar). Se realizó análisis univariado y bivariado y se midió la fuerza de asociación calculando odds ratio, con intervalos de confianza del $95 \%$ y la prueba $x 2$ para buscar el valor de $p$.

Resultados. El 79,1 \% de los casos presentaron estrés, 71,48 \% depresión y 73,49\% ansiedad. Todos estos factores se comportaron como factores de riesgo para la enfermedad (OR = 8,54; IC $95 \%: 5,8-12,45 ; p=0,000)(O R=5,76 ;$ IC $95 \%: 4,07-8,17 ; p=0,000)$ (OR $=6,25$; IC $95 \%: 4,4-8,91 ; p=0,000)$ respectivamente, al igual que la disfuncionalidad familiar, el no soporte de amigos y los hábitos sociales como el café (OR $=1,44$; IC $95 \%$ : $1,04-1,99 ; p=0,02),(O R=1,67$; IC $95 \%: 1,21-2,31 ; p=0,001)(O R=1,78 ;$ IC $95 \%$ : $1,29-2,44 ; p=0,002)$ respectivamente.

Conclusiones. Factores psicosociales como la depresión, ansiedad y estrés son factores de riesgo que predisponen a desarrollar o complicar el Síndrome de Intestino Irritable. Palabras clave. Síndrome de Intestino Irritable, Ansiedad, Depresión, Estrés.

\section{ABSTRACT}

Psychosocial, sociodemographic, cultural and family factors associated with Irritable Bowel Syndrome

Objective. To describe the psychosocial factors, demographic, cultural and family associated with irritable bowel syndrome in adults.

Method. Case-control study in 754 patients, 256 diagnosed with Irritable Bowel Syndrome (cases) and 498 patients without disease (controls) between 30 and 60 years in three private health institutions Cartagena randomly selected, non should be cognitively impaired, diagnosis of cancer of the colon, stomach, pancreas, rectum or suffer from gastro intestinal tract.

Measurements. We filled out a questionnaire that included the test Golberg (anxiety and depression), social adjustment scale of Holmes and Rahe (stress) and the Family APGAR (family functioning). Was performed univariate and bivariate analysis was measured by calculating the strength of association Odd ratio, confidence intervals $95 \%, x 2$ to find the value of $p$.

Results. $79.1 \%$ of cases had stress, $71.48 \%$ depression and $73.49 \%$ anxiety. All these factors acted as risk factors for disease $(\mathrm{OR}=8,54$; $95 \% \mathrm{Cl} 5,8-12,45, \mathrm{p}=0,000)(\mathrm{OR}=$ 5,76; $95 \% \mathrm{Cl} 4,07-8,17 ; p=0,000)(\mathrm{OR}=6,25 ; 95 \% \mathrm{Cl} 4,4-8,91 ; p=0,000)$ respectively, as dysfunctional families, friends do not support and the social habits such as coffee (OR = 1,44; $95 \% \mathrm{Cl}: 1,04-1,99, \mathrm{p}=0,02)(\mathrm{OR}=1,67 ; 95 \% \mathrm{Cl} 1,21-2,31 ; \mathrm{p}=0,001)(\mathrm{OR}=$ $1,78 ; 95 \% \mathrm{Cl} 1,29-2,44 ; \mathrm{p}=0,002)$ respectively.

Conclusions. Psychosocial factors such as depression, anxiety and stress are risk factors that predispose to or complicate develop Irritable Bowel Syndrome.

Key words. Irritable Bowel Syndrome, Anxiety, Depression, Stress. 


\section{INTRODUCCIÓN}

El síndrome de intestino irritable (SII) es una enfermedad crónica y recidivante que se caracteriza por dolor o malestar abdominal con alteración del hábito intestinal como diarrea o estreñimiento ${ }^{1} \mathrm{y}$ forma parte de la categorización de los criterios ROMA III. Debido a su alta prevalencia, entre el 4 y $20 \%$ a nivel mundial, se considera una enfermedad de gran impacto en la calidad de vida ${ }^{2,3}$. En países industrializados se observa una prevalencia del 10 al $15 \%$ \% ${ }^{4,5}$. En Estados Unidos es una de las causas más frecuentes de absentismo laboral después de la gripe $^{2}$ y genera 2,4 a 3,5 millones de consultas médicas y más de dos millones de prescripciones, produciendo costos superiores a 33 mil millones de dólares ${ }^{2}$. En los países Latinoamericanos como Brasil, México, Uruguay y Colombia se observa una prevalencia entre el 9 y $18 \%$ \%,5,6.

Su etiología y factores asociados continúan siendo poco claros, lo que ha estimulado a muchas investigaciones. Los síntomas depresivo-ansiosos como el estrés psicosocial ${ }^{7,8}$ son relacionados con la enfermedad; otros agentes que podrían constituirse en factor de riesgo lo conforman los aspectos sociodemográficos, como la edad ${ }^{2}$, sexo ${ }^{2,6,9}$, ocupación ${ }^{10}$ y estado civil ${ }^{10}$, los socio culturales ${ }^{10}$ ,como los hábitos de consumo de alcohol, café y tabaco, y aspectos relacionados con la dinámica y funcionalidad familiar ${ }^{11}$. El objetivo de este estudio es conocer cómo estos factores se relacionan con el Síndrome de Intestino Irritable con el objeto de mejorar los métodos de diagnóstico y controlar las hiperconsultas y el sobrecosto que permita elaborar e instaurar un programa de atención para alcanzar dicho objetivo.

\section{MATERIAL Y MÉTODO}

Se realizó un estudio de casos y controles, entre diciembre de 2006 y marzo de 2007 en pacientes adultos de tres instituciones privadas prestadoras de salud de Cartagena. Se obtuvo una muestra de 754 (256 casos y 498 controles) para una exposición de los factores de riesgo estudiados en los controles del $10 \%$, un nivel de confianza del $95 \%$, una potencia del $80 \%$ y una relación caso control de 1:2. Los sujetos de estudio se seleccionaron por muestreo aleatorio simple cumpliendo los siguientes requisitos: para los casos, que tuviesen diagnostico de Síndrome de Intestino Irritable, edad de 30 a 60 años y el consentimiento de participar en el estudio; y para los controles los mismos criterios, pero sin el diagnostico de la enfermedad. Ambos grupos no debían presentar discapacidad cognitiva, diagnostico de cáncer de colon, gástrico, páncreas, recto ni padecer alteraciones del TGI.
A través de la historia clínica y entrevista se recolectó la información, que fue registrada en un instrumento previamente validado que incluía las variables sociodemográficas (edad y sexo), familiares (tipo de familia y el APGAR Familiar para medir funcionalidad familiar y soporte de amigos), hábitos sociales (consumo de café, alcohol y tabaco), psicosociales (test de Goldberg para medir depresión y ansiedad, y escala de reajuste social de Holmes y Rahe para medir estrés). A partir de los datos, utilizando el Software Epi-info versión 3.3.2, se realizó la estadística descriptiva y se midió la fuerza de asociación calculando Odds Ratio, con intervalos de confianza del $95 \%$. Se utilizó la prueba $X^{2}$ como medida de significación estadística para buscar el valor de $\mathrm{p}$ y determinar si las diferencias encontradas en casos y controles eran estadísticamente significativas.

\section{RESULTADOS}

Se encontraron los siguientes resultados al relacionarse el Síndrome Intestino Irritable con:

- Factores sociodemográficos: se encontró que el pertenecer al sexo femenino (OR = 1,92; IC 95 \%: $1,35-2,72 ; p=0,0001)$ y al estrato socioeconómico bajo (OR = 1,69; IC $95 \%$ : 1,02-2,81; $p=$ $0,03)$ significó un riesgo para presentar la enfermedad. Las edades más tempranas, entre los 30 a 45 años se comportaron como factor protector $(\mathrm{OR}=0,62$; IC 95 \%: 0,44-0,88; $\mathrm{p}=0.005)$ (tabla 1).

- Factores familiares: La disfuncionalidad familiar se comportó como un factor de riesgo (OR = 1,44; IC 95 \%: 1,04-1,99; $p<0,01$ ) para la aparición de la enfermedad, pero, al contrario de esto, el pertenecer a una familia nuclear sería un factor protector contra la enfermedad (OR $=0,51$; IC 95 \%: 0,34-0,75; $p=0,0000$ ). En aquellas personas que no vivían con sus familiares el no presentar soporte de amigos se comportó como un factor de riesgo para la enfermedad $(\mathrm{OR}=1,67$; IC 95 \%: 1,21-2,31; $p<0,01$ ) (tabla 1).

- Factores culturales: el consumir café antes de ser diagnosticado el Síndrome de Intestino Irritable fue el único hábito que se comportó como factor de riesgo para desarrollar la enfermedad $(\mathrm{OR}=$ 1,78; IC 95 \%: 1,29-2,44; $p=0,002$ ) (tabla 1).

- Factores psicosociales: tanto el estrés, como la depresión y la ansiedad, se comportaron como factores de riesgo ante la enfermedad, arrojando valores de $p=0,00000$ (tabla 1 ). 


\begin{tabular}{|c|c|c|c|c|c|}
\hline VARIABLE & CASOS & CONTROLES & OR & IC $95 \%$ & $\mathbf{P}$ \\
\hline \multicolumn{6}{|l|}{ Edad } \\
\hline $30-45$ & 165 & 378 & & & \\
\hline $46-60$ & 84 & 120 & 0,62 & $0,44-0,88$ & 0,005 \\
\hline \multicolumn{6}{|c|}{ Estrato Socioeconómico } \\
\hline Bajo $(1,2,3)$ & 224 & 419 & & & \\
\hline Alto $(4,5)$ & 25 & 79 & 1,69 & $1,02-2,81$ & 0,03 \\
\hline \multicolumn{6}{|l|}{ Estado Civil } \\
\hline Con Pareja & 114 & 254 & & & \\
\hline Sin Pareja & 135 & 244 & 0,81 & $0,59-1,11$ & 0,178 \\
\hline \multicolumn{6}{|l|}{ Sexo } \\
\hline Femenino & 186 & 302 & & & \\
\hline Masculino & 63 & 196 & 1,92 & $1,35-2,72$ & 0,0001 \\
\hline \multicolumn{6}{|l|}{ Ocupación } \\
\hline Ama de Casa & 74 & 126 & & & \\
\hline Otras Profesiones & 175 & 372 & 1,25 & $0,88-1,78$ & 0,19 \\
\hline \multicolumn{6}{|l|}{ Tipo de Familia } \\
\hline No Nuclear & 45 & 151 & & & \\
\hline Nuclear & 204 & 347 & 0,51 & $0,34-0,75$ & 0,0000 \\
\hline \multicolumn{6}{|c|}{ Antecedentes de Tabaco } \\
\hline $\mathrm{Si}$ & 55 & 84 & & & \\
\hline No & 194 & 414 & 1,40 & $0,90-2,08$ & 0,083 \\
\hline \multicolumn{6}{|c|}{ Consumo Actual de Tabaco } \\
\hline $\mathrm{Si}$ & 41 & 96 & & & \\
\hline No & 208 & 412 & 0,83 & $0,54-1,26$ & 0,349 \\
\hline \multicolumn{6}{|c|}{ Antecedentes de Alcohol } \\
\hline $\mathrm{Si}$ & 112 & 227 & & & \\
\hline No & 137 & 271 & 0,98 & $0,71-1,34$ & 0,087 \\
\hline \multicolumn{6}{|c|}{ Consumo Actual de Alcohol } \\
\hline $\mathrm{Si}$ & 93 & 239 & & & \\
\hline No & 156 & 259 & 0,65 & $0,65-0,47$ & 0,005 \\
\hline \multicolumn{6}{|c|}{ Antecedentes de Café } \\
\hline $\mathrm{Si}$ & 144 & 217 & & & \\
\hline No & 105 & 281 & 1,78 & $1,29-2,44$ & 0,002 \\
\hline \multicolumn{6}{|c|}{ Consumo Actual de Café } \\
\hline $\mathrm{Si}$ & 116 & 215 & & & \\
\hline No & 133 & 283 & 1,15 & $0,84-1,58$ & 0,37 \\
\hline \multicolumn{6}{|l|}{ Estrés } \\
\hline $\mathrm{Si}$ & 197 & 153 & & & \\
\hline No & 52 & 345 & 8,54 & $5,8-12,45$ & 0,000000 \\
\hline \multicolumn{6}{|l|}{ Depresión } \\
\hline $\mathrm{Si}$ & 178 & 151 & & & \\
\hline No & 71 & 347 & 5,76 & $4,07-8,17$ & 0,000000 \\
\hline \multicolumn{6}{|l|}{ Ansiedad } \\
\hline $\mathrm{Si}$ & 183 & 153 & & & \\
\hline No & 66 & 345 & 6,25 & $4,4-8,91$ & 0,000000 \\
\hline \multicolumn{6}{|c|}{ Funcionalidad Familiar } \\
\hline Disfuncional & 157 & 270 & & & \\
\hline Funcional & 92 & 228 & 1,44 & $1,04-1,99$ & 0,02 \\
\hline \multicolumn{6}{|l|}{ Soporte de Amigos } \\
\hline No Presenta & 157 & 270 & & & \\
\hline Si Presenta & 92 & 228 & 1,67 & $1,21-2,31$ & 0,0012 \\
\hline
\end{tabular}

Tabla 1. Factores psicosociales, sociodemográficos, culturales y familiares asociados a Síndrome de Intestino Irritable en pacientes que asisten a tres Instituciones de Salud de Cartagena. 


\section{DISCUSIÓN}

El Síndrome de Intestino Irritable es más frecuente en mujeres con una relación de 3:1 con respecto a los hombres ${ }^{9}$. En este estudio las mujeres también fueron más frecuentes en una relación de 2:1 con respecto a los hombres. En lo referente al estrato socioeconómico, se encontró que esta variable podía convertirse en un factor de riesgo, siendo los estratos socioeconómicos 1, 2, 3, considerados de clase baja y media baja, los más afectados con intestino irritable. Ayala y col. ${ }^{11}$ encontraron altas prevalencias de Síndrome de Intestino Irritable en nivel socioeconómico bajo.

El consumo de sustancias como el café y el alcohol podrían intervenir de forma importante en el progreso de la patología asociados a la exacerbación de las crisis o periodos sintomáticos de la enfermedad $^{12}$. El hábito del café en este estudio se comportó como un factor de riesgo para la enfermedad, lo que no sucedió con otros hábitos como el consumo de alcohol y tabaco.

Existen varios estudios de investigadores psiquiátricos que mostraron asociación de Intestino Irritable y depresión en la población general ${ }^{13,14}$. Hislop encontró relación entre el síndrome y depresión en ambientes clínicos ambulatorios ${ }^{13}$. Campos y col. ${ }^{14}$ encontraron una prevalencia de síntomas gastrointestinales bajos del $26 \%$ y de síntomas depresivos del $29,7 \%$ en pacientes psiquiátricos, a diferencia de la gran prevalencia presentada por este estudio, donde el $71,48 \%$ de los pacientes con intestino irritable sufrían de depresión.

Valdría la pena discutir la fuerte asociación encontrada entre la presencia de ansiedad e intestino irritable, ya que coincidimos con esta asociación, como la encontrada por Word, que reportó que el $26 \%$ de 200 pacientes con criterios diagnósticos de síndrome de pánico y ansiedad experimentó síntomas digestivos significativos, especialmente vómitos y diarrea ${ }^{15}$. Lydiard describió que el $42 \%$ de los pacientes consecutivos admitidos con síndrome de pánico describieron espontáneamente síntomas gastroenterológicos como su molestia más importante, durante entrevistas estructuradas utilizando los criterios del DSM-III para síndrome de pánico (el cual no discrimina síntomas digestivos en la entrevista). De éstos, el $12 \%$ estaban en tratamiento con gastroenterólogos por síndrome de intestino irritable. El tratamiento farmacológico exitoso de su síndrome de pánico coincidió con la resolución de su cuadro intestinal ${ }^{16}$.

El estrés también se comportó como un factor de riesgo, al igual que lo reportado por Saíto, donde los pacientes con Intestino Irritable solían acudir al médico luego de un problema de estrés, depresión, pérdida laboral o algún otro evento estresante ${ }^{2}$. Otra característica de los pacientes con esta enfermedad es que a menudo relatan situaciones de estrés inmediatamente antes de que se instaure el trastorno. Esto ha llevado a investigar la prevalencia de sucesos vitales estresantes. Más del $50 \%$ relaciona la aparición de sus síntomas con la vivencia de algún suceso estresante tal como dificultad en el trabajo, muerte de algún familiar, intervenciones quirúrgicas, problemas de pareja o historia de abuso sexual, entre otros ${ }^{17}$. Pero de la misma forma podemos deducir que la disfuncionalidad familiar y el no soporte de amigos, al ser asociados con el síndrome, podrían convertirse en un factor de riesgo para sufrir de intestino irritable, al igual que el tipo de familia no nuclear, ya que estas condiciones podrían generar algún tipo de estrés en el individuo y complicar la sintomatología de esta enfermedad.

Es así cómo los resultados de este estudio y otras publicaciones aquí citadas evidencian que una vez más los factores psicosociales como la depresión, ansiedad y estrés son factores de riesgo que predisponen a desarrollar o complicar el Síndrome de Intestino Irritable. Ello indica que al estado emocional de los pacientes con intestino irritable debería dedicársele cada vez mayor atención para garantizar la salud integral de la población, oportuna y eficaz. La aparición y complicaciones del síndrome de intestino irritable puede también estar asociadas a variables sociodemográficas como la edad, el sexo y el estrato socioeconómico.

\section{BIBLIOGRAFÍA}

1. Olden KW, Drossman DA. Psychological and psychiatric aspects of gastrointestinal disease. Med Clin North Am. 2000; 84:1313-27.

2. Saito YA, Schoenfeld P, Locke GR. The epidemiology of irritable bowel syndrome in North America: A systematic review. Am J Gastroenterology. 2002; 97:1910-5.

3. Gomez D, Rojas L, Morales J, Mujica S. Síndrome de Intestino irritable: Una perspectiva actualizada. MedUNAB. 2008; 11:50-60.

4. Valenzuela J, Alvarado J, Cohen H, Damiao A, Francisconi $C$, Frugone L, et al. Un consenso Latinoamericano sobre el síndrome del intestino irritable. Gastroenterol Hepatol. 2004; 27:325-43.

5. Ángel LA, Amaya A, Perilla C, Góngora A, Sánchez A, Bohórquez $C$, et al. Prevalencia del síndrome de intestino irritable y factores asociados. Acta Méd Colomb. 1997; 22:219-24.

6. Torres DP. Epidemiología del síndrome de intestino irritable. Consenso del síndrome de intestino irritable. Rev Colomb Gastroenterol. 2003; 18:8-11.

7. Farthing MJG. Treatment options in irritable bowel syndrome. Best Pract Res Clin Gastroenterology. 2004; 18:773-86.

8. Masand PS, Kaplan DS, Gupta S, Bhandary AN, Nasra GS, Kline MD, et al. Major depression and irritable bowel 
syndrome: is there a relationship? J Clin Psychiatry. 1995; 56:363-7.

9. Drossman DA, Camilleri M, Mayer EA, Whitehead WE. AGA Technical review on irritable bowel syndrome. Gastroenterology. 2002; 123:2108-31.

10. Gaburri M, Bassotti G, Bacci G, Cinti A, Bosso R, Ceccarelli $\mathrm{P}$, et al. Functional gut disorders and health care seeking behavior in an Italian non-patient population. Recent Prog Med. 1989; 80:241-4.

11. Smilkstein G, Ashworth C, Montano D. Validity and reliability of the Family APGAR as a test of family function. J Fam Pract. 1982; 15:303-11.

12. El-Serag HB, Olden K, Bjorkman D. Health-related quality of life among persons with irritable bowel syndrome: a systematic review. Aliment Pharmacol Ther. 2002; 16:1171-85.
13. Hislop IG. Effect of very brief psychotherapy on the irritable bowel síndrome. Med J Aust. 1980; 2:620-3.

14. Smilkstein G. The family APGAR: a proposal for a family function test and its use by physicians. J Fam Pract. 1978; 6:1231-9.

15. Wood JD. Neuropathophysiology of irritable bowel syndrome. J Clin Gastroenterol. 2002; 35:11-22.

16. Lydiard RB, Laraia MT, Howell EF, Ballenger JC. Can panic disorder present as irritable bowel syndrome? J Clin Psychiatry. 1986; 47:470-3.

17. Creed F, Craig T, Farmer R. Functional abdominal pain, psychiatric illness, and life events. Gut 1988; 29:235-42. 